\title{
Composición de la dieta de otoño del pato mexicano (Anas diazi) en el vaso sur de las ciénegas del Lerma, Estado de México
}

\author{
Fall diet composition of Mexican duck (Anas diazi) at Lerma marsh, South Vessel, México \\ State
}

\author{
David Colón-Quezada
}

Laboratorio de Zoología, Facultad de Ciencias, Universidad Autónoma del Estado de México.

Instituto Literario 100, Col. Centro, 50000, Toluca, Estado de México, México.

Correspondencia: colon_qd@yahoo.com.mx

\begin{abstract}
Resumen. Se estudió la composición de la dieta del pato mexicano (Anas diazi) y su variación en 2 periodos de otoño consecutivos (2004-2005) en el vaso sur de las ciénegas del Lerma, Estado de México, a partir de 24 muestras de patos cazados. El análisis complementario del peso seco agregado y la frecuencia mostraron que durante el otoño el pato mexicano tiene una dieta vegetariana en la que predominan las semillas de plantas acuáticas, malezas, maíz y tubérculos de Sagittaria y un mínimo consumo de invertebrados. Los alimentos que principalmente se consumieron en 2004 fueron semillas de Echinichloa holciformis, Polygonum lapathifolium y P. punctatum, en tanto que en la dieta de 2005, fueron semillas de maíz y tubérculos de Sagittaria macrophylla. El análisis de similitud de dietas de Sorensen, evidenció una variación de $70 \%$ entre los 2 años, lo cual puede atribuirse a la disponibilidad anual de alimento.
\end{abstract}

Palabras clave: Anas diazi, hábitos alimenticios, semillas, malezas, Echinichloa holciformis, Polygonum, maíz, Sagittaria macrophylla.

\begin{abstract}
The Mexican duck's diet composition and its variation in 2 consecutive fall periods (2004-2005) was studied at the Lerma marsh, South Basin, Mexico State, including 24 esophageal samples obtained during the hunting season. A complementary analysis using aggregated dry weight and frequency of occurrence showed than the Mexican duck has a vegetarian diet dominated by aquatic plants seeds, weeds seeds, corn seeds and Sagittaria tubes. The main foods in 2004 were Echinochloa holciformis, Polygonum lapathifolium and P. punctatum seeds, whereas corn seeds and Sagittaria macrophylla tubers dominated the 2005 diet. The Sorensen similarity analysis of the diets showed a diet variation of $70 \%$ on both years, which could be to attributed to annual food availability.
\end{abstract}

Key words: Anas diazi, food habits, seeds, weeds, Echinichloa holciformis, Polygonum, corn, Sagittaria macrophylla.

\section{Introducción}

El pato mexicano, Anas diazi (Escalante et al., 1996), es un anatido residente mexicano catalogado por la norma oficial mexicana NOM-059-ECOL-2001 como especie amenazada (SEMARNAT, 2002); se ubica en la tribu Anatini que corresponde a los patos de superficie, es decir, los que forrajean alcanzando el fondo de los humedales por inclinación, o bien, internándose superficialmente en la columna de agua (Johnsgard, 1978; Bellrose, 1980; Krapu y Reinecke, 1992). Esta especie se distribuye desde el eje neovolcánico transversal en los estados de México, Tlaxcala, Puebla y Distrito Federal, siguiendo a través de la meseta central en Zacatecas, Guanajuato, Michoacán,

Recibido: 06 julio 2007; aceptado: 29 agosto 2008 hacia el noroeste en Nayarit y Jalisco, al norte en los estados de Chihuahua y Durango, y hasta el sureste de Arizona y el valle del río Grande al norte de Albuquerque, Nuevo Mexico en los Estados Unidos (Leopold, 1959; Aldrich y Baer, 1970; Bellrose, 1980; Saunders y Saunders, 1981). En el Estado de México, las ciénegas de la cuenca alta del río Lerma son un importante hábitat de residencia para esta especie, encontrándose tanto grupos invernantes como reproductivos (Saunders y Saunders, 1981).

En las ciénegas del río Lerma, como en la mayoría de sus sitios de distribución en México, los estudios sobre el pato mexicano son escasos. La información que existe gira en torno a aspectos morfológicos (Scott y Reynolds, 1984), taxonómicos (Pitelka, 1948; Johnsgard, 1961; Aldrich y Baer 1970), poblacionales (Pérez-Artega et al., 2002) y recientemente de uso de hábitat (Medina et al., 2007), 
pero se carece de investigación sobre su alimentación, la cual, no obstante, se asocia con el consumo de semillas directamente de los campos de cultivo. En la mayoría de los casos, lo anterior se deriva tanto de observaciones fortuitas en campo de patos mexicanos alimentándose en cultivos de maíz, trigo, arroz y garbanzo, como del análisis de algunas muestras de esófagos de patos mexicanos cazados, en las que se han encontrado dichas semillas como el alimento que consumen preferentemente (Leopold, 1959). Sin embargo, estos datos adolecen de falta de carácter científico, por lo que el panorama que ofrecen acerca de los hábitos alimenticios de la especie y de las condiciones ecológicas implicadas es limitado.

Los hábitos alimenticios invernales de patos migratorios de superficie se han estudiado con amplitud en Estados Unidos, y en casos excepcionales en México. Los resultados de dichos estudios revelan que durante el periodo invernal (otoño-invierno) los patos adoptan una dieta basada en el consumo preponderante de semillas de hidrófitas emergentes, sumergidas y/o semillas cultivadas. McGilvrey (1966) registró que en lago Marion, Carolina del Sur, las semillas de diversas especies de plantas constituyeron el $96 \%$ en la dieta de otoño del pato de collar (Anas platyrhynchos), el $98.6 \%$ en la del pato calvo (Anas americana), el 99\% en la del golondrino (Anas acuta), el $97 \%$ en la del negro (Anas rubripes) y el $85 \%$ en el cerceta alas verdes (Anas crecca). De forma análoga, Miller (1987) encontró que el 98\% de la dieta de otoño del pato golondrino en el valle de Sacramento en California, se compone de semillas de por lo menos 13 especies, incluyendo arroz (Oriza sativa), como especie cultivada y $2 \%$ de invertebrados. Por otro lado, Migoya y Baldassarre (1993) informan que en la bahía de Santa María, Sinaloa, México, las semillas de hidrófitas, principalmente de tule (Scyrpus maritimus), dominan en la dieta de invierno del cerceta alas verdes (79.8\%), del pato golondrino (90.6\%), del cerceta canela (Anas cyanoptera) $(66.6 \%)$ y del pato cuchara (Anas clypeata) (62.8\%), mientras que los invertebrados conforman el $20.2 \%, 9.4 \%, 33.4 \%$ y $37.2 \%$ de la dieta, respectivamente. En fecha reciente, Colón et al. (2007) mencionan que la dieta invernal del cerceta alas azules (Anas discors) en la laguna de Chiconahuapan en el vaso sur de las ciénegas del Lerma se compone del $80 \%$ de semillas y $20 \%$ de invertebrados.

Desde el punto de vista ecológico, el estudio de los hábitos alimenticios es una herramienta importante en la formulación de programas de manejo y conservación, en particular si se desea mejorar la calidad y cantidad de recursos en los hábitats de las aves en los sitios de invernación, migración y reproducción, ya que permite conocer las necesidades nutricionales y ambientales de las especies, así como las infecciones parasitarias y la bioacumulación de contaminantes que están directamente relacionados con la ingesta de alimentos (Bartonek y Hickey, 1969; Dirschl, 1969; Krapu et al., 1973; Austin et al., 1999). En este sentido, el propósito de este estudio fue conocer la dieta del pato mexicano y su variación en 2 periodos de otoño (2004-2005) en el vaso sur de las ciénegas del Lerma, que sea información puntual para el manejo del hábitat en el corto y mediano plazo.

\section{Materiales y métodos}

Área de estudio. La colecta de muestras se llevó a cabo en el vaso sur o laguna de Chignahupan $\left(19^{\circ} 09^{\prime} 05^{\prime \prime} \mathrm{N}\right.$, 99 30’13”'O, 2570 m snm), que se localiza en los municipios de Almoloya del Río y Texcalyacac, Estado de México (Fig. 1). El humedal tiene una extensión de 700 ha, y está ubicado en el sur del complejo de humedales conocidos como Ciénegas del Lerma, decretadas Áreas Naturales Protegidas (SEMARNAT, 2002a), hábitat prioritario para aves acuáticas residentes y migratorias (Ramsar list, 2006) y Áreas de Importancia para la Conservación de las Aves (Arizmendi y Márquez, 2000).

Aproximadamente, el $30 \%$ de la superficie del cuerpo acuático es agua abierta con una profundidad promedio de $70 \mathrm{~cm}(40 \mathrm{~cm}-150 \mathrm{~cm})$. El $70 \%$ de la superficie restante, con profundidad promedio de $50 \mathrm{~cm}$ (30-120 $\mathrm{cm}$ ), está cubierta por vegetación hidrófita emergente en la que predominan especies como el tule redondo (Schoenoplectus tabernaemontani; Cyperaceae), tule palmilla (Typha latifolia; Typhaceae) y el tule esquinado (Schoenoplectus americanus, Cyperaceae). De acuerdo con la zonación de lagos y lagunas de Arriaga Cabrera et al. (2000), en la zona litoral la vegetación dominante la componen especies anuales de ciperáceas como Eleocharis macrostachya y Cyperus sp., algunas especies de chilillo (Polygonum punctatum, P. lapathifolium y P. mexicanum; Polygonaceae), Leersia hexandra (Poaceae), Echinochloa sp., papa de agua (Sagittaria latifolia; Alismataceae), papa de aplacol (S. macrophylla; Alismataceae), varias especies de juncáceas. En los canales y zanjas se encuentran especies como berro palmilla (Berula erecta; Apiaceae), berro de hoja ancha (Hydrocotyle ranunculoides; Apiaceae).

En la actualidad, la vegetación acuática sumergida se restringe a áreas pequeñas en las orillas del humedal o en canales y zanjas alrededor de éste, donde se pueden encontrar cola de caballo (Myriophyllum aquaticum; Haloragaceae), bejuquillo (Ceratophyllum sp.; Ceratophyllaceae), Chiara sp., cebolleja (Lilaea scilloides; Juncaginaceae), grama (Potamogeton pectinatus; Potamogetonaceae) y hierba de agua (Potamogeton ilinoensis; Potamogetonaceae). La vegetación libre flotadora la componen tamborcillo 


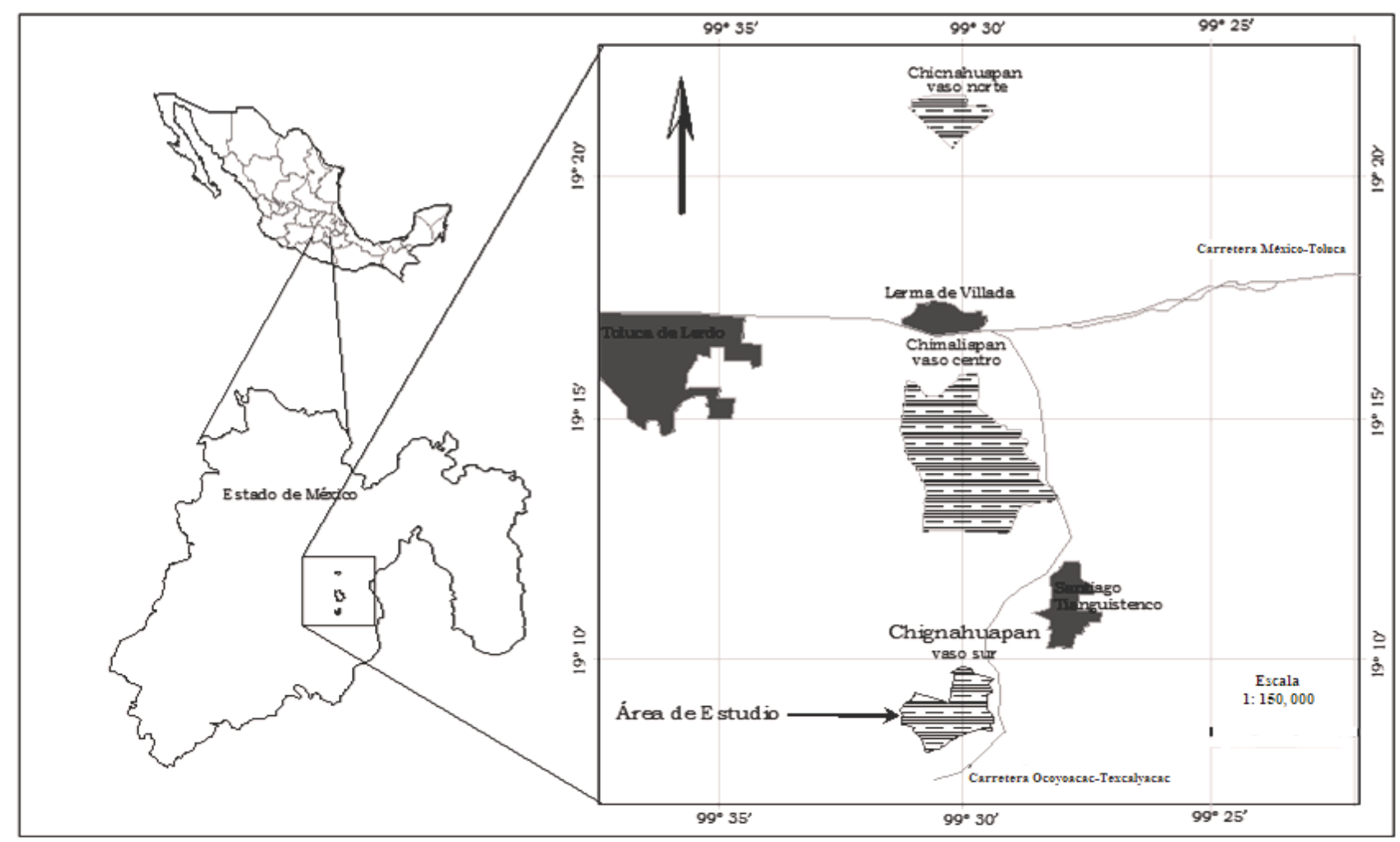

Figura 1. Ubicación del área de estudio. Tomado y modificado de Ciénegas del Lerma, sitio RAMSAR 1335 en México, Comisión Nacional de Áreas Naturales Protegidas.

(Hydromystria laevigata; Hydrocharitaceae), helecho de agua (Azolla filiculoides; Salviniaceae), utricularias (Utricularia sp.; Lentibulariaceae), y algunas lentejillas de agua (Lemnaceae), como Lemna gibba, Lemna trisulca, Wolfiella lingulata, Wolffiella oblonga, Wolffia columbiana.

Análisis de muestras: se tomaron muestras del contenido alimenticio del esófago y proventrículo de patos mexicanos donados por cazadores en el periodo del 7 de noviembre al 24 de diciembre de 2004 y 2005. Las muestras se tomaron de patos recién cobrados que tuvieran hasta 2 horas post mortem, con la finalidad de obtener muestras lo menos deterioradas por efecto de la digestión post mortem, y que fueran un reflejo fehaciente de todos los alimentos consumidos. De cada muestra se removió el contenido alimenticio del esófago y proventrículo, colocándose en frascos de plástico con etanol al 80\% para evitar la digestión post mortem (Swanson y Bartonek, 1970). El alimento se clasificó, se identificó taxonómicamente y se midió con base en su biomasa (peso seco), secándose en una estufa a masa constante a $65^{\circ} \mathrm{C}$ (Combs y Frederickson, 1996), temperatura óptima probada para la deshidratación de las semillas de maíz y tubérculos, que fueron los recursos de mayor tamaño y con mayor contenido de agua. En la mayoría de los casos, la identificación de los alimentos vegetales se hizo hasta especie, con base en Martin y Barkley (1961), Correl y Correl (1972), González (1985) y Espinosa y Sarukhán (1997). Los alimentos de origen animal se identificaron a orden y en algunos casos se determinó la familia y especie, con base en Pennak (1978) y Merrit y Cummins (1996).

Para reducir el efecto de alimentos de consumo infrecuente con un alto porcentaje de peso seco agregado en la dieta, la composición de la dieta se determinó mediante el análisis complementario del método del porcentaje de peso seco agregado de cada alimento y la frecuencia con la que cada alimento se presentó en el total de aves de la muestra (Swanson et al., 1974; Krapu y Reinecke, 1992; Custer y Custer, 1996):

Porcentaje de peso seco agregado=

Proporción promedio de cada alimento en cada ave

Total aves

Frecuencia $=$ No de aves en las que se encontró " $X$ " alimento

Total de aves 
Se obtuvo el porcentaje de similitud de las dietas de los 2 años con el coeficiente de similitud de Sorensen (Greig-Smith en: Dirchl 1969):

$$
\frac{\text { (2c) } 100}{a+b}
$$

donde $a$ : número de alimentos en el primer año

$b$ : número de alimentos en el primer año $c$ : número de alimentos en común

\section{Resultados}

En 2004, el total de muestras fue de 15 y en 2005, de 9. En noviembre de 2004 se obtuvieron 6 y en diciembre 9; 13 correspondieron a machos y 2 a hembras. En noviembre de 2005 se obtuvieron 6 muestras y 3 en diciembre; 3 correspondieron a machos y 6 a hembras. Puesto que el número de muestras por mes fue pequeño, se agruparon en una sola muestra por año, abarcando de la mitad al final del otoño ( 7 de noviembre a 24 de diciembre). Debido a que fue poco el número de muestras de hembras en 2004 $(\mathrm{n}=2)$, al igual que el número total de muestras en 2005. No se realizaron análisis estadísticos ni estimaciones de valores promedio de los alimentos consumidos por hembras o machos para cada año con objeto de determinar diferencias intersexuales en la dieta, para evitar incurrir en sobrestimaciones que desvirtuaran los resultados.

Se identificaron 17 taxones de alimento vegetal y 9 de alimento animal. El alimento vegetal comprendió 10 familias, 15 géneros y 17 especies. Predominaron las familias Gramineae, Polygonaceae y Alismataceae; los géneros Echinochloa, Zea, Polygonum y Sagitaria, y las especies Echinochloa holciformis, Zea mays, Polygonum punctatum, P. laphatifolium y Sagittaria macrophylla. Del alimento de origen animal, Mollusca, Anisoptera y Hemiptera fueron los órdenes más importantes.

En los 2 periodos de otoño estudiados, la dieta se compuso principalmente de alimentos vegetales; y las semillas de plantas acuáticas, maíz, malezas, además de tubérculos de papas de agua (Sagittaria sp.) conformaron más del $99 \%$ del peso seco agregado de la dieta. El consumo de invertebrados fue reducido, cubriendo menos del $1 \%$ de la dieta en ambos años (Fig. 2).

En la dieta del otoño de 2004 dominaron principalmente las semillas de plantas acuáticas que se desarrollan en la zona litoral del humedal y en los campos de cultivo aledaños a éste. Los alimentos más importantes en la dieta fueron semillas de cebadilla (Echinochloa holciformis), Polygonum lapathifolium y $P$. punctatum que en conjunto constituyeron el $76.17 \%$ de la dieta (Cuadro 1). Aun cuando el porcentaje de peso seco agregado de cada especie muestra una ligera diferencia en el consumo entre
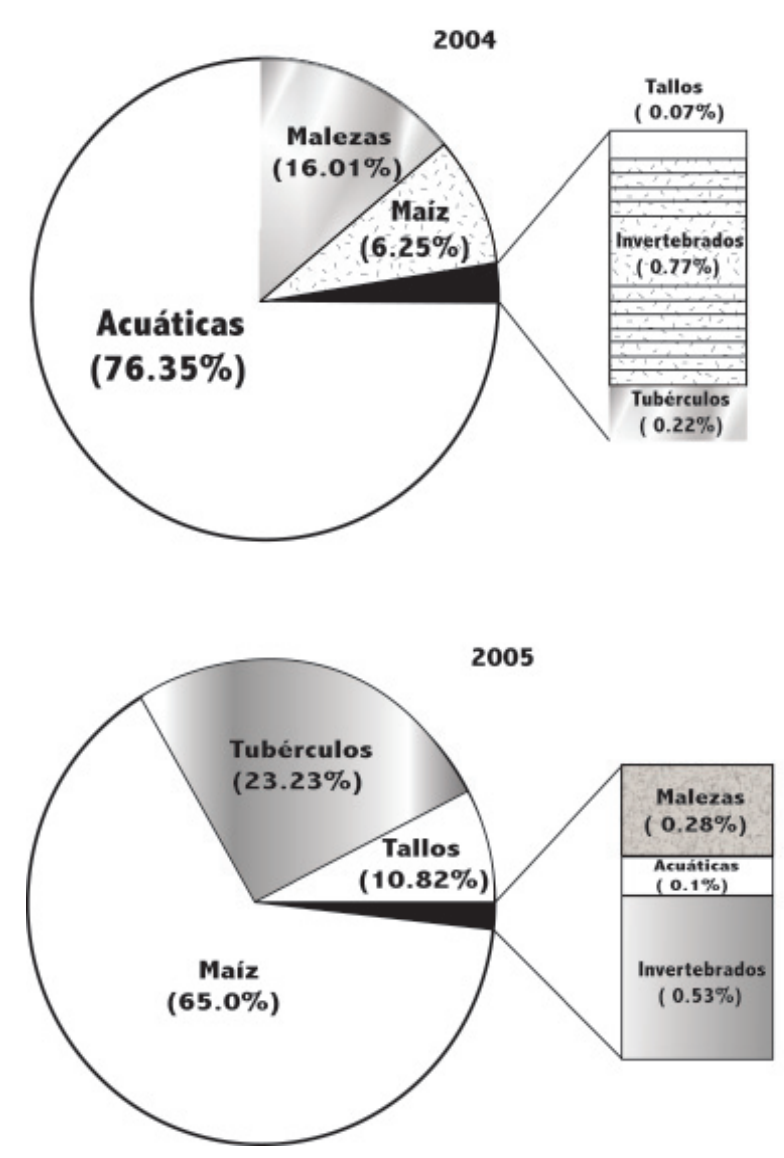

Figura 2. Composición de la dieta de patos mexicanos en el otoño de 2004 y de 2005.

especies, con la aplicación de un análisis de varianza, no se detectaron diferencias significativas en el consumo de masa seca agregada entre las 3 especies de plantas $(\mathrm{F}=$ 0.40, D.F. 2, $\mathrm{p}=0.674$ ).

Adicionalmente, se identificó una miscelánea de semillas, de las cuales las más importantes fueron una dicotiledónea de la familia Malvaceae no identificada, Tripogandra purpurascens, Echinopepeon milleflorus, maíz (Zea mays) y Tinantia erecta, que en conjunto conformaron el $21.95 \%$ del peso seco agregado de la dieta. El resto de la materia vegetal, el $0.61 \%$ de la dieta, consistió en un combinado de semillas (Fig. 2).

Los invertebrados constituyeron el $0.77 \%$ de la dieta, siendo los gasterópodos y anisópteros los mejor representados, ambos con una proporción del peso seco agregado $<0.5 \%$ de la dieta (Cuadro 1). Otros invertebrados fueron sanguijuelas (Hirudinea), larvas de moscos (Ephidridae), larvas de escarabajos acuáticos (Hydrophilidae) y un escarabajo acuático adulto del género Tropisternus. 
Cuadro 1. Composición de la dieta de otoño de 2004 de patos mexicanos en las ciénegas del sur de la cuenca alta del río Lerma. (A), acuática (C), cultivada (M), maleza

\begin{tabular}{|c|c|c|c|}
\hline Alimento & \multicolumn{2}{|c|}{$\%$ Peso seco agregado $(g)$} & Presencia $(\%)(n=15)$ \\
\hline \multirow[t]{2}{*}{ Alimento vegetal (semillas) } & \multicolumn{2}{|c|}{98.90} & \\
\hline & $\bar{x}$ & Intervalo & \\
\hline \multicolumn{4}{|l|}{ Gramineae } \\
\hline Echinochloa holciformis (A) & 27.28 & $0.1-94.5$ & 80.0 \\
\hline Zea mays (C) & 6.25 & $0.0-93.8$ & 6.6 \\
\hline \multicolumn{4}{|l|}{ Polygonaceae } \\
\hline Polygonum lapathifolium (A) & 24.64 & $0.7-98.7$ & 80.0 \\
\hline Polygonum puctatum (A) & 24.25 & $0.8-99.5$ & 53.3 \\
\hline \multicolumn{4}{|l|}{ Malvaceae (M) } \\
\hline Especie no identificada & 9.4 & $0.7-67.7$ & 33.3 \\
\hline \multicolumn{4}{|l|}{ Commelinaceae } \\
\hline Tripogandra purpurascens (M) & 2.12 & $0.07-25.2$ & 33.3 \\
\hline Tinantia erecta $(\mathrm{M})$ & 3.12 & $0.0-46.6$ & 6.6 \\
\hline \multicolumn{4}{|l|}{ Cucurbitaceae } \\
\hline Echinopepon milleflorus (M) & 1.19 & $0.2-17.2$ & 20.0 \\
\hline \multicolumn{4}{|l|}{ Cyperaceae } \\
\hline Eleocharis sp. (A) & 0.15 & $0.2-1.02$ & 20.0 \\
\hline Tallos de Eleocharis sp (A) & 0.07 & $0.0-0.07$ & 6.6 \\
\hline \multicolumn{4}{|l|}{ Compositae } \\
\hline Conyza sp (M) & 0.04 & $0.2-0-5$ & 13.3 \\
\hline \multicolumn{4}{|l|}{ Apiaceae } \\
\hline Hydrocotyle ranunculoides (A) & 0.03 & $0.0-0.01$ & 6.6 \\
\hline \multicolumn{4}{|l|}{ Alismataceae } \\
\hline Sagittaria macrophylla (tubérculos)(A) & 0.22 & $0.0-3.1$ & 6.6 \\
\hline \multicolumn{4}{|l|}{ Caryophyllaceae } \\
\hline Sagina procumbens $(\mathrm{M})$ & 0.10 & $0.0-1.6$ & 6.6 \\
\hline Alimento animal & & 77 & \\
\hline Physa sp (Mollusca; Physidae) & 0.28 & $0.11-1.7$ & 53.3 \\
\hline Anisopteros (Larvas) & 0.34 & $0.11-3.4$ & 20.0 \\
\hline Hirudineos (Hirudinea) & 0.04 & $0.11-0.3$ & 13.3 \\
\hline $\begin{array}{l}\text { Tropisternus } s p \\
\quad \text { (Coleoptera, Hydrophilidae adulto) }\end{array}$ & 0.02 & $0.0-0.1$ & 6.6 \\
\hline Hydrophilidae (Coleoptera, larvas) & 0.04 & $0.0-0.5$ & 6.6 \\
\hline Ephidridae (Diptera, larvas) & 0.05 & $0.0-0.8$ & 6.6 \\
\hline
\end{tabular}

El 65\% de la dieta de otoño de 2005 lo conformaron semillas de maíz seguido por tubérculos de Sagittaria macrophylla con el $12 \%$ de la dieta (Cuadro 2). Se identificaron otros 7 alimentos de origen vegetal, presentándose uno por muestra. Estos alimentos generalmente estuvieron en proporción mucho menor en muestras donde dominaron las semillas de maíz y los tubérculos de $S$. macrophylla, con excepción de los tubérculos de Sagittaria latifolia y tallos de Eleocharis sp., que aportaron más del $10 \%$ cada uno, pero sólo en una muestra, respectivamente, por lo que no se consideran importantes en la dieta.

Los invertebrados conformaron el $0.52 \%$ del peso seco agregado, siendo los coríxidos (Hemiptera) el grupo de 
Cuadro 2. Composición de la dieta de otoño de 2005 de patos mexicanos en las ciénegas del sur de la cuenca alta del río Lerma. (A), acuática (C), cultivada (M), maleza

\begin{tabular}{|c|c|c|c|}
\hline \multirow{3}{*}{$\begin{array}{r}\text { Alimento } \\
\text { Alimento vegetal (semillas) }\end{array}$} & \multirow{2}{*}{\multicolumn{2}{|c|}{$\begin{array}{c}\% \text { Peso seco agregado (g) } \\
99.43\end{array}$}} & \multirow[t]{3}{*}{ Presencia $(\%)(n=9)$} \\
\hline & & & \\
\hline & $\bar{x}$ & Intervalo & \\
\hline \multicolumn{4}{|l|}{ Gramineae } \\
\hline Zea mays (C) & 65.00 & $89.3-100$ & 66.6 \\
\hline \multicolumn{4}{|l|}{ Alismataceae } \\
\hline Sagittaria macrophylla (tubérculos)(A) & 12.36 & $1.6-99.4$ & 33.3 \\
\hline Sagittaria latifolia (Tubérculos) (A) & 10.87 & $0.0-97.8$ & 11.1 \\
\hline \multicolumn{4}{|l|}{ Cyperaceae } \\
\hline Tallos de Eleocharis sp (A) & 10.82 & $0.0-97.4$ & 11.1 \\
\hline Eleocharis sp. (A) & 0.07 & $0.0-0.6$ & 11.1 \\
\hline Schoenoplectus tabernaemontani (A) & 0.01 & $0.0-0.1$ & 11.1 \\
\hline \multicolumn{4}{|l|}{ Polygonaceae } \\
\hline Polygonum lapathifolium (A) & 0.02 & $0.0-0.19$ & 11.1 \\
\hline Rumex crispus $(\mathrm{M})$ & 0.27 & $0.0-2.4$ & 11.1 \\
\hline \multicolumn{4}{|l|}{ Gramineae } \\
\hline Pоа аппиа $(\mathrm{M})$ & 0.01 & $0.0-0.1$ & 11.1 \\
\hline$\underline{\text { Alimento animal }}$ & & & \\
\hline Corixidae (Hemiptera, adultos) & 0.13 & $0.5-0.6$ & 22.2 \\
\hline Gammaridae (Anfípoda, Crustacea) & 0.28 & $0.0-2.54$ & 11.1 \\
\hline Cambarellus montezumae (Crustacea) & 0.09 & $0.0-0.8$ & 11.1 \\
\hline Hydrophilidae (Coleoptera, larvas) & 0.03 & $0.0-0.3$ & 11.1 \\
\hline
\end{tabular}

mayor frecuencia (Cuadro 2).

El consumo de las semillas de maíz y tubérculos de Sagittaria macrophylla tuvo un desarrollo antagónico en la composición de la dieta a lo largo del otoño (Fig. 3). Las semillas de maíz fueron el principal alimento en la dieta $(\bar{x}=99.4 \%$, rango 99.1-99.8\%) del 13 al 21 de noviembre; a partir de esa fecha el detrimento fue continuo hasta el 19 de diciembre en que nuevamente incidieron en las muestras. A su vez, el consumo de tubérculos de $S$. macrophylla estuvo confinado a las últimas 3 semanas del otoño, en las que junto con los de $S$. latifolia fueron constituyendo progresivamente un mayor porcentaje en la dieta $(\bar{x}=75 \%$, rango $52.4-97.8 \%)$ (Fig. 3$)$.

Contrario a lo que se hubiera esperado, la composición de la dieta en ambos años fue diametralmente diferente. Mientras que en la dieta del otoño de 2004 predominaron las semillas de plantas acuáticas, en la del 2005 fueron las de maíz y los tubérculos de Sagittaria macrophylla, sin incidencia prominente de malezas, conservando el $37.5 \%$ de similitud entre ellas, siendo más variada la dieta del 2004 (Cuadro 1 y Cuadro 2).

\section{Discusión}

Los hábitos alimenticios en ambos periodos de otoño evidenciaron una alimentación predominantemente vegetariana a base de semillas y tubérculos, y un consumo mínimo de invertebrados acuáticos, manifestando que el alimento vegetal es la principal fuente nutricional y energética en el otoño. Si bien el uso de muestras de aves cazadas podría predisponer los resultados hacia una presencia alta de alimentos vegetales (Swanson y Bartonek, 1970), el uso de muestras de esófagos, provee una imagen real de los alimentos consumidos por los patos mexicanos al tener una baja tasa de digestión (Dirschl, 1969). Así pues, la dominancia de semillas en la dieta de los patos mexicanos es consistente con los hábitos alimenticios de otoño de otros patos de superficie, como la cerceta alas verdes (Euliss y Harris, 1987; Burns et al., 2003), el pato golondrino (Euliss y Harris, 1987; Miller, 1987; Burns et al., 2003), el pato de collar (Burns et al., 2003) o el pato negro (McGilvrey, 1966) que consumen principalmente semillas de plantas acuáticas o semillas 


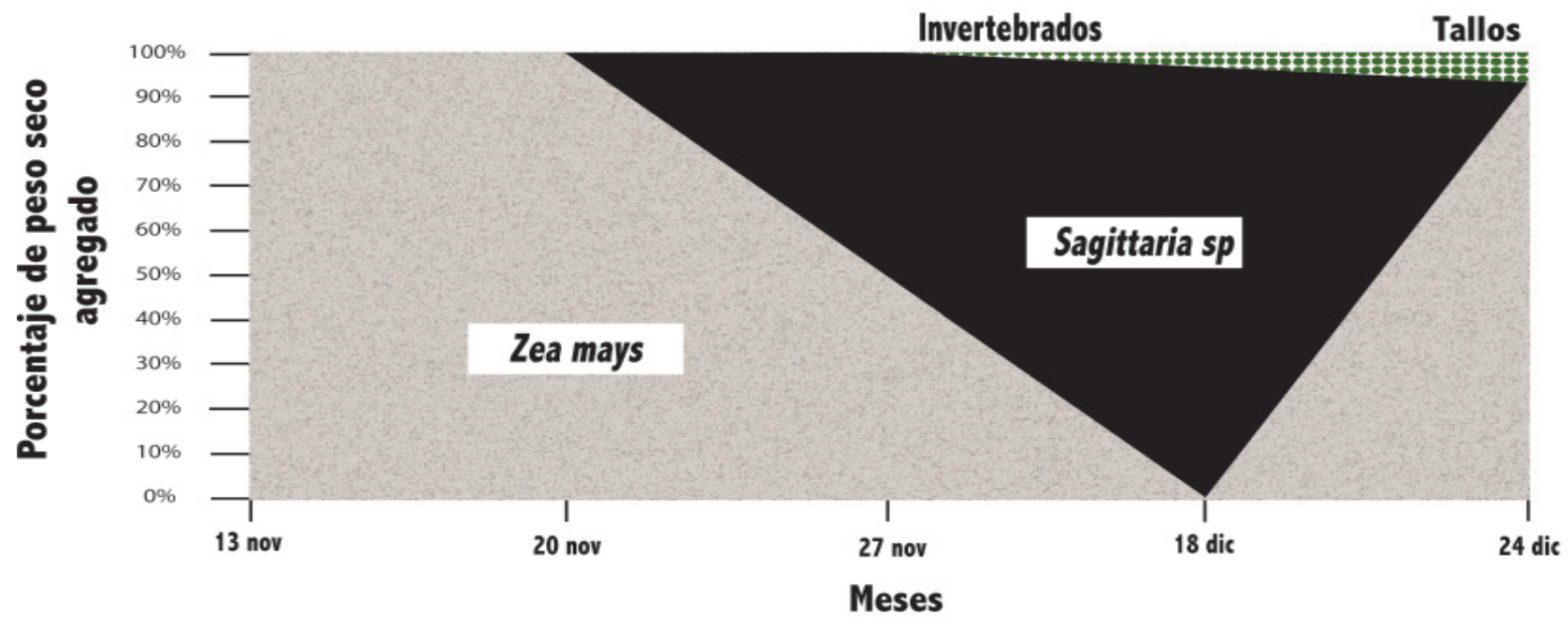

Figura 3. Variación temporal en el consumo de semillas de maíz y tubérculos de Sagittaria sp. en otoño de 2005.

de plantas cultivadas. El consumo preponderante de semillas en la dieta invernal es una conducta asociada a la necesidad de conseguir alimentos ricos en carbohidratos, imprescindibles para solventar las necesidades termofisiológicas de la temporada, dado el aporte inmediato de alta energía que proporcionan, indispensable para mitigar el estrés térmico de la temporada (Jorde y Owen, 1988).

En este estudio, los principales alimentos fueron esencialmente especies vegetales anuales propias de la zona litoral del humedal, que también se desarrollan en los campos de cultivo contiguos que otrora formaban parte del humedal, y que al anegarse se convierten en importantes áreas de alimentación, incrementando la variedad de alimentos en la dieta como ocurrió en 2004.

Las semillas de cebadilla, $P$. lapathifolium y $P$. punctatum fueron los principales alimentos en la dieta del pato en 2004, semejante a lo que registran Colón et al. (2007) para el cerceta alas azules en la misma área de estudio. Ellos encontraron que las semillas de Echinochloa sp. fueron el principal alimento en la dieta de otoño de 2004 e invierno de 2005, en tanto que las de Polygonum punctatum fueron el segundo en importancia en el invierno. La similitud de los resultados en los 2 estudios, aunada a los altos valores de frecuencia de la cebadilla $(80 \%), P$. lapathifolium (80\%) y P. punctatum (50\%) en la dieta de otoño del pato mexicano, indica que estas especies fueron probablemente las más abundantes y altamente disponibles, explicando en parte su dominancia.

La cebadilla y las 2 especies de Polygonum se desarrollan en algunos sitios de zona litoral del humedal, como canales y zanjas someras, y en los que delimitan los predios anexos al humedal, aunque son más abundantes en los campos de cultivo de maíz y haba (Vicia faba), en los que la cebadilla sólo aparece cuando están anegados, dado su carácter acuático (González, 1985). Derivado del análisis de la composición de alimentos en cada muestra se encontró que en el $66 \%$ de las muestras en las que predominaron las semillas de Polygonum, su consumo estuvo acompañado por la incidencia de semillas de Tinantia erecta, Echinopepon milleflorus, Tripogandra purpurascens y/o la malvacea no identificada, que son especies arvenses (Espinosa y Sarukán, 1997; Villaseñor Ríos y Espinosa García, 1998; Vieyra-Odilon y Vibrans, 2001) características en los cultivos de maíz y haba aledaños al humedal, siendo ésta una evidencia indirecta de que los patos mexicanos utilizaron campos de cultivo como áreas de alimentación durante el otoño, lo que pone de manifiesto que al menos en 2004, la alimentación en campos de cultivo se orientó al aprovechamiento de semillas de plantas acuáticas y malezas que en estos se desarrollan, más que al consumo de semillas cultivadas. Bossenmaier y Marshall (en Baldassarre y Bolen, 1994) y Bellrose (1980) mencionan una situación similar en el pato de collar que prefiere los alimentos naturales que el maíz, aún cuando esté disponible en campos de cultivo, lo cual se atribuye al hecho de que los alimentos naturales proveen a los patos de una dieta nutritivamente más completa que el maíz (Baldassarre et al., 1983). Así pues, la alimentación en campos de cultivo fortalece la idea de la adaptabilidad del pato mexicano a los ambientes agrícolas, aprovechando los recursos que éstos proveen temporalmente como una posible respuesta a la pérdida de humedales naturales y a la falta de alimento, producto de la degradación continua a la que están sometidos los humedales, como también lo han expresado Scott y Reynolds (1984) y Pérez-Artega et al. (2002). 
Con referencia a lo anterior, se observó que P. punctatum y P. lapathifolium pueden aparecer de manera abundante en campos de cultivo de habas, en ocasiones establecidos en zonas dominadas por pastos en la zona litoral del humedal. En abril cuando dichas zonas están completamente secas, algunas superficies se preparan para el cultivo de habas mediante la remoción del suelo, lo que trae consigo la germinación de abundantes plantas de Polygonum sp., y malezas como Tinantia erecta, Echinopepon milleflorus, Tripogandra purpurascens. Dichas superficies, de anegarse en el verano, se ha visto que son áreas de alimentación para patos residentes y migratorios durante el otoño e invierno. Estos hechos vislumbran la posibilidad de comenzar a manejar las superficies dominadas por pastos en la zona litoral del humedal para convertirlas en áreas de alimentación a través de la remoción mecánica del suelo, asegurando su anegación con la construcción de diques o canales.

En general, el consumo de semillas de Echinochloa sp. y Polygonum sp., no dista de lo que se ha registrado para algunos patos de superficie en Norteamérica (v. gr. cerceta alas verdes, Bellrose, 1980; golondrinos, Yocom, 1951), pues durante el otoño e invierno las semillas de varias especies de Echinochloa y Polygonum son ampliamente consumidas por los patos que frecuentan los límites de los cuerpos de agua donde estas plantas abundan (Correl y Correl, 1972; Baldassarre y Bolen, 1994), sugiriendo que las semillas de estos géneros son alimentos naturales comunes en la dieta invernal de los anátidos de superficie en diferentes latitudes geográficas.

En la dieta de 2005, las semillas de maíz y los tubérculos de Sagittaria macrophylla fueron los alimentos más importantes. En lo que se refiere al maíz, que es el principal cultivo en los terrenos agrícolas contiguos al humedal, su incidencia al parecer estuvo relacionada con la provisión humana premeditada de estas semillas. Esto se deduce del hecho de que en 2005 los campos de maíz no se anegaron, por lo que se cree que las semillas de maíz consumidas, procedían de espacios de agua abierta destinados a la actividad cinegética en el interior del humedal, donde los organizadores cinegéticos agregan maíz para cebar a los patos, al menos una vez por semana, o bien, de las orillas del humedal donde personas residentes de las comunidades aledañas las depositaban para atraer patos y gallaretas (Fulica americana) que capturaban con trampas rústicas para venderlos precocidos en algunos tianguis en el valle de Toluca (obs. pers). No obstante, el alto consumo de semillas de maíz también ha sido documentado en el pato de collar por Bellrose (1980), Jorde et al. (1983) y Baldassarre y Bolen (1984), quienes refieren que cuando los alimentos naturales de los humedales son escasos y limitadamente disponibles, los patos de collar basan su alimentación en el consumo de semillas de maíz en campos de cultivo cosechados, no anegados, llegando a conformar entre el 60 y $100 \%$ de su dieta invernal. Esta conducta es una estrategia exitosa de forrajeo que presumiblemente han desarrollado los patos como respuesta a la reducción de alimentos naturales debida a la pérdida y degradación de los humedales o cuando el hielo cubre la superficie y disminuye la disponibilidad de los recursos. Es posible que esta situación haya ocurrido en el presente estudio, pues en la dieta de 2005 se registró un menor número de alimentos consumidos, particularmente de semillas de plantas acuáticas, respecto a la dieta de 2004, lo que conduce a suponer que el consumo de semillas de maíz pudo obedecer a la aparente escasez de alimento natural, por lo que sitios cebados con maíz pudieron ser altamente atractivos para los patos mexicanos, sin descartar su posible consumo en campos de cultivo no anegados.

Es presumible que el consumo de tubérculos de Sagittaria hacia el final del otoño fue precedido por la fenología de las plantas, ligado a la disminución tanto de la temperatura ambiental como de la profundidad de la columna de agua. Zepeda (2001) y Zepeda y Lot (2005) refieren que la mayor disponibilidad de tubérculos comienza a partir de noviembre cuando el nivel del agua disminuye y las primeras heladas del año producen la muerte de las plantas y la degeneración de los estolones, dejando libres los tubérculos bajo los sedimentos. Por tanto, se infiere que la conjugación de dichos factores favoreció la aparición de este recurso en la dieta hacia el final del otoño, llegando a ser tan altamente aprovechado que en un solo pato se encontraron 173 tubérculos de S. macrophylla con un diámetro promedio de $0.71 \mathrm{~cm}$. La relación trófica entre el pato mexicano y los tubérculos de $S$. macrophylla adquiere una alta trascendencia ecológica al tratarse de 2 especies endémicas mexicanas amenazadas de extinción, según la NOM-059-ECOL-2001, únicas en la cuenca del río Lerma y del Valle de México, donde $S$. macrophylla se distribuye exclusivamente (Novelo y Lot, 1990; Haynes y Holm-Nielsen, 1994). Esto otorga un valor agregado para la conservación de los hábitats donde ambas especies cohabitan.

La discrepancia en la composición de la dieta entre los 2 periodos de otoño, sugiere que la disponibilidad de alimento ligada a la variación anual de las condiciones climáticas, particularmente de precipitación, fue quizá el factor ecológico que rigió la dieta de un año a otro favoreciendo la germinación de semillas así como la anegación de los campos de cultivo aledaños al humedal permitiendo el consumo de las semillas de las malezas que en estos se desarrollan, como se supone ocurrió en 2004. Un ejemplo de dicha situación pudo haberse reflejado en el aprovechamiento tanto de las semillas de Polygonum como 
las de cebadilla en ambos años, ya que aún cuando las plantas de Polygonum pueden desarrollarse prescindiendo de la anegación (Ramos-Ventura, 2000), -ya sea en las orillas del humedal como en los campos de cultivo-, su consumo está sujeto a la acumulación de agua temporal o estacional que permite a los patos filtrar las semillas acumuladas en los sedimentos. Una situación similar se presenta en el caso de la cebadilla, ya que al ser una especie acuática (González, 1985; Ramos-Ventura, 2000), su desarrollo, y por ende su aprovechamiento, dependen completamente de la anegación.

La variedad de alimentos consumidos en los 2 periodos de otoño estudiados, refleja la plasticidad del pato mexicano para explotar diversos alimentos acorde con las condiciones del hábitat, lo que resulta importante, porque al no ser especialista, para el manejo de sus hábitats se cuenta con un amplio abanico de estrategias a utilizar en torno a la restauración ecológica con especies de menester alimenticio para la especie, preferentemente de gramíneas, polygonaceas y alismataceas. Finalmente, dada la naturaleza y disposición de los alimentos consumidos se infiere que esta especie, al menos durante el otoño, tiene hábitos de forrajeo bentónicos apegados a la zona litoral del humedal y en campos de cultivo anegados, por lo que los sitios de baja profundidad son una necesidad ecológica importante para su alimentación.

\section{Agradecimientos}

A la M. en C. Blanca Jaimes Cruz (Laboratorio de Zoología, Facultad de Ciencias, Universidad Autónoma del Estado de México), por las facilidades otorgadas para el análisis de las muestras; la M. en C. Carmen Zepeda Gómez (Laboratorio Ecología de Plantas Acuáticas, Facultad de Ciencias, Universidad Autónoma del Estado de México), por su apoyo en la determinación taxonómica de las semillas y plantas; la Biól. Josefina Curiel Álvarez, por su ayuda en la determinación de los insectos e invertebrados; la Dra. Heike Vibrans (Laboratorio de Etnobotánica, Colegio de Postgraduados), en la determinación de semillas; el Biól. Antonio Soto Méndez, por su apoyo en la toma de muestras; la Secretaría del Medio Ambiente y Recursos Naturales (SEMARNAT), delegación Edo. de México; el Biól. Pedro E. Díaz Díaz, responsable técnico del área de estudio, por las facilidades para la toma de muestras, y el Geog. Noé A. Aguirre González, por la elaboración del mapa de localización.

\section{Literatura citada}

Aldrich, J. W. y K. P. Baer. 1970. Status and speciation in the mexican duck (Anas diazi). Wilson Bulletin 82:63-73.
Arizmendi, M. C. y L. Márquez Valdemar. 2000. Áreas de importancia para la conservación de las aves en México. Sección Mexicana del Consejo Internacional para la Preservación de las Aves (CIPAMEX). México, D.F. 440 p.

Arriaga Cabrera. L., V. Aguilar Sierra y J. Alcocer Durand. 2000. Aguas continentales y diversidad biológica de México. Comisión Nacional para el Conocimiento y Uso de la Biodiversidad. México, D. F. 327p.

Austin, J. E., A. D. Afton, M. G. Anderson, R. G. Clark, C. C. Custer, J. S. Lawrence, J. B. Pollard y J. K. Ringleman. 1999. Declines of greater and lesser scaup populations: issues, hypotheses, and research directions. Summary report for the scaup workshop. U.S. Geological Survey, Northern Prairie Wildlife Research Center. Jamestown, North Dakota. 18 p.

Baldassarre, G. A., R. J. Whyte, E. E. Quinlan y E. G. Bolen. 1983. Dynamics and quality of waste corn available to postbreeding waterfowl in Texas. Wildlife Society Bulletin 11:25-31

Baldassarre, G. A. y E. G. Bolen. 1984. Field-feeding ecology of waterfowl wintering on the southern high plains of Texas. Journal of Wildlife Management 48:63-71.

Baldassarre, G. A. y E. G. Bolen. 1994. Waterfowl ecology and management. Wiley, New York. 609 p.

Bartonek, J. C. y J. J. Hickey. 1969. Food habits of canvasbacks, redhead, and lesser scaup in Manitoba. Condor 71:280-290.

Bellrose, F. C. 1980. Ducks, gesse and swans of North America, tercera edición Stackpole, Books, Harrisburg, Pennsylvania. $40 \mathrm{p}$.

Burns, E., M. Miller y J. Eadie. 2003. An analysis of food habits of green-winged teal, northern pintails and mallards wintering in Suisin marsh to develop guidelines for food plant management. A report to the California Department of Water Resources and the United States Bureau of Reclamation, California. $66 \mathrm{p}$.

Colón, Q. D., G. A. Cavazos y V. E. Maldonado. 2007. Dieta invernal de la cerceta alas azules (Anas discors) en las Ciénegas del Lerma. Memorias del VII Congreso para el Estudio y Conservación de las Aves en México, Sección Mexicana del Consejo Internacional para la Preservación de las Aves (CIPAMEX). San Francisco de Campeche, octubre de 2007.

Combs, D. L. y L. H. Fredrickson. 1996. Foods used by male mallards wintering in southeaestern Missouri. Journal of Wildlife Management 60:603-610.

Correl, D. S. y H. B. Correl. 1972. Aquatic and wetland plants of southwestern United States. Stanford Univesity Press, California. $1777 \mathrm{p}$.

Custer, C. M. y T. W. Custer. 1996. Food habits of diving ducks in the Great Lakes after the zebra mussel invasion. Journal of Field Ornithology 67:86-99.

Dirschl, H. J. 1969. Foods of lesser scaup and blue-winged teal in the Saskatchewan river Delta. Journal of Wildlife Management 33:77-87.

Escalante, P., A. M. Sada y J. R. Gil. 1996. Listado de nombres comunes de las aves de México. Comisión Nacional para el Conocimiento y Uso de la Biodiversidad/Sierra Madre., México, D. F. 32 p. 
Espinosa, G. F. J. y J. Sarukhán. 1997. Manual de malezas del valle de México. Fondo de Cultura Económica/Universidad Nacional Autónoma de México, México,D. F. 407 p.

Euliss, N. H. Jr. y S. W. Harris. 1987. Feeding ecology of northern pintails and green-winged teal wintering in California. Journal of Wildlife Management 51:724-732.

González, R. A. 1985. Las gramíneas lacustres y palustres del Valle de México. Tesis de Licenciatura. Facultad de Ciencias, Universidad Nacional Autónoma de México, México, D. F. $101 \mathrm{p}$.

Haynes, R. R. y L. B. Holm-Nielsen. 1994. The Alismataceae. Flora Neotropica. Mon. 64:1-112.

Johnsgard, P. A. 1961. Evolutionary relathionships among the North American mallards. Auk 78:3-43.

Johnsgard, P.A. 1978. Ducks, geese, and swans of the world. University of Nebraska Press, Lincoln. 404 p.

Jorde, D. G., G. L. Krapu y R. D. Crawford. 1983. Feeding ecology of mallards wintering in Nebraska. Journal of Wildlife Management 47:1044-1053

Jorde, D. G. y R. B. Owen Jr. 1988. Efficienciy of nutrient use by American black ducks wintering in Maine. Journal of Wildlife Management 52:209-214.

Krapu, G. L., G. A. Swanson y H. K. Nelson. 1973. Mercury residues in pintails breeding in North Dakota. Journal of Wildlife Management 37:395-399.

Krapu, G. L. y K. J. Reinecke. 1992. Foraging ecology and nutrition. In Ecology and management of breeding waterfowl, B. D. J. Batt, A. D. Afton, M. G. Anderson, C. D. Ankney, D. H. Hohnson, J. A. Kadlec y G. L. Krapu (eds.). University of Minnesota Press, Minneapolis. p. 1-29.

Leopold, A. S. 1959. Wildlife of Mexico. University of California Press, Berkeley. 568 p.

McGilvrey, B. F. 1966. Fall food habits of ducks near Santee refuge, South Carolina. Journal of Wildlife Management 30:577-580.

Martin, A. C. y W. D. Barkley. 1961. Seed identification manual. University of California Press, Berkeley. 221 p.

Medina, T. S. M., O. M. Márquez y M. E. García. 2007. Uso y selección de embalses por el pato mexicano (Anas diazi) en la región del Llano, Aguascalientes-Jalisco, México. Acta Zoológica Mexicana 23:163-181.

Merrit, R. W. y K. W. Cummins. 1996. An introduction to the aquatic insects of North America, tercera edición, Kendall/ Hunt, Dubuque, Iowa. 862 p.

Migoya, R. y G. A. Baldassarre. 1993. Harvest and food habits of waterfowl wintering in Sinaloa, Mexico. The Southwestern Naturalist 38:168-171.

Miller, R. M. 1987. Fall and winter foods of northern pintails in the Sacramento Valley, California. Journal of Wildlife Management 51:405-414

Novelo, A. y A. Lot. 1990. Alismataceae. In Flora Fanerogámica del Valle de México, vol. III, J. Rzedowski y G. C. de Rzedowski Instituto de Ecología, Pátzcuaro, Michoacán. p. 26-30.

Pennak, R. W. 1978. Fresh-water invertebrates of the United Status, segunda edición, Wiley New York. 803 p.

Pérez-Arteaga, A., J. K. Gaston y M. Kershaw. 2002. Population trends and priority conservation sites for Mexican duck Anas diazi. Bird Conservation International 12:35-52

Pitelka, F. A. 1948. Notes on the distribution and taxonomy of Mexican game birds. Condor 50:113-123

Ramsar list. 2006. The list of wetlands of international importance. Convention on Wetlands of Internacional Importance (Ramsar, Iran, 1971). Ramsar Convention, Gland. 1-38 p.

Ramos-Ventura, L. J. 2000. Estudio de la flora y vegetación acuáticas vasculares de la Cuenca Alta del río Lerma, en el Estado de México. Tesis, Maestría Facultad de Ciencias, UNAM, México. 146 p.

Saunders, G. B. y D. C. Saunders. 1981. Waterfowl and their wintering grounds in Mexico, 1937-64. Washington, D.C.: U.S. Fish and Wildlife Service (resource publication 138). $151 \mathrm{p}$.

SEMARNAT (Secretaría de Medio Ambiente y Recursos Naturales). 2002. Norma Oficial Mexicana NOM-059ECOL-2001. Protección ambiental-Especies nativas de México de flora y fauna silvestres-Categorías de riesgo y especificaciones para su inclusión, exclusión o cambio-Lista de especies en riesgo. Diario Oficial de la Federación, 6 de marzo de 2002, México, D.F. p. 1-85.

SEMARNAT (Secretaría de Medio Ambiente y Recursos Naturales). 2002a. Decreto por el que se declara área natural protegida, con el carácter de área de protección de flora y fauna, la región conocida como Ciénegas del Lerma, ubicada en los municipios de Lerma, Santiago Tianguistenco, Almoloya del Río, Capulhuac, San Mateo Atenco, Metepec y Texcalyacac en el Estado de México, con una superficie total de 3,023-95-74.005 hectáreas. Diario Oficial de la Federación, 26 de noviembre de 2002, México, D.F. p. 4-12.

Scott, N. J., Jr. y R. P. Reynolds. 1984. Phenotipic variation of the Mexican duck (Anas platyrhynchos diazi) in Mexico. Condor 86:266-274

Swanson, G. A. y J. C. Bartonek. 1970. Bias associated with food analysis in gizzards of blue-winged teal. Journal of Wildlife Management 34:739-746

Swanson, G. A., G. L. Krapu, J. C. Bartonek, J. R. Serie y D. H. Johnson. 1974. Advantages in mathematically weighting waterfowl food habits data. Journal of Wildlife Management 38:302-307.

Vieyra-Odilon, L. y H. Vibrans. 2001. Weeds as crops: the value of maize field weeds in the Valley of Toluca, Mexico. Economic Botany 55:426-443.

Villaseñor Ríos, J. L. y F. J. Espinosa García. 1998. Catálogo de malezas de México. Universidad Nacional Autónoma de México/Consejo Nacional Consultivo Fitosanitario/ Fondo de Cultura Económica, México, D.F. 448 p.

Yocom, C. F. 1951. Waterfowl and their foods plants in Washington. University of Washington Press, Seattle. 272 p.

Zepeda, C. 2001. Distribución, caracterización ecológica y etnobotánica de Sagittaria macrophylla y S. latifolia, en la cuenca alta del río Lerma, Estado de México. Tesis Maestría. Facultad de Ciencias, UNAM, México, D. F. 91 p.

Zepeda, C. y A. Lot. 2005. Distribución y uso tradicional de Sagittaria macrophylla Zucc. y S. latifolia Willd. en el Estado de México. Ciencia ergo sum (12-3):282-290. 